\title{
Droit naturel et histoire chez François Gény
}

Nader Hakim

(c) https://publications-prairial.fr/cliothemis/index.php?id=1534

DOI : 10.35562/cliothemis.1534

Référence électronique

Nader Hakim, « Droit naturel et histoire chez François Gény », Clio@Themis [En ligne], 9 | 2015, mis en ligne le 24 juin 2021, consulté le 15 septembre 2021. URL: https://publications-prairial.fr/cliothemis/index.php?id=1534

Droits d'auteur

CC BY-NC-SA 


\title{
Droit naturel et histoire chez François Gény
}

\author{
Nader Hakim
}

\section{PLAN}

I. Histoire et immuabilité du droit

II. Histoire et évolutions de la société

\section{TEXTE}

1 La présente étude n'entend pas revenir sur la vie et l'œuvre complète du doyen nancéien dorénavant assez bien connues ${ }^{1}$, mais seulement explorer la relation qu'entretient le célèbre auteur de Méthode d'interprétation ${ }^{2}$ et de Science et technique ${ }^{3}$ avec l'histoire en général, et l'histoire du droit en particulier. Cet aspect de l'œuvre de François Gény (1861-1959) n'a été que très peu abordé ${ }^{4}$ alors qu'il nous semble susceptible de permettre de mieux comprendre tant la pensée du civiliste que le rôle ou le recours à l'histoire au sein de la doctrine juridique contemporaine.

2 S'il relève certes de l'évidence que le professeur de Nancy n'est pas historien et que l'histoire n'est pas au centre de ses préoccupations, ne serait-ce qu'en le comparant à son mentor Raymond Saleilles ${ }^{5}$, il n'en demeure pas moins qu'il ne peut faire abstraction ni de la méthode historique alors en vogue, ni de l'importance de l'histoire dans la science et la culture de son temps. À la suite de Portalis, il reconnaît ainsi que l'histoire est « la physique expérimentale de la jurisprudence [...] un vaste champ d'expériences (les seules expériences pratiquement concevables en matière sociale), et que nous devons consulter, bien moins comme une révélation de la vérité en soi (qui peut fort bien avoir été méconnue dans les faits), que comme un pierre de touche, éprouvant le mérite des systèmes, dont elle nous retrace et l'origine et les destinées ». Il ajoute d'ailleurs que cette vérité scientifique profonde et durable a été affirmée par l'École historique allemande car « les règles juridiques sont le produit de forces et de besoins immanents aux rapports de la vie, et [...] l'étude approfondie de l'histoire permet seule de découvrir ces forces, comme de 
peser ces besoins ». Ainsi reconnaît-il que l'histoire a bien une valeur éducatrice car « une étude pénétrante du passé fait seule bien saisir les problèmes de l'heure présente ${ }^{6}$.

Si l'on ne peut pas douter de la sincérité de ces déclarations de principe, la question de savoir si elles emportent de réels choix méthodologiques demeure pleine et entière. Or, nous pouvons en douter ne serait-ce qu'en comparant Gény et deux de ses benjamins pourtant très proches de ses convictions les plus fortes. À l'inverse d'Eugène Gaudemet $^{7}$ ou de Julien Bonnecase ${ }^{8}$ en effet, le doyen de Nancy n'éprouve nul besoin de faire l'histoire de ses prédécesseurs pour critiquer leur méthode ${ }^{9}$. Il peut se contenter d'analyser leurs idées et d'en faire une critique intellectuelle décontextualisée et presque totalement déhistoricisée ${ }^{10}$.

4 Gény diverge également de certains de ses contemporains ${ }^{11}$, et surtout de ses prédécesseurs civilistes du $\mathrm{xIX}^{\mathrm{e}}$ siècle dans la fonction qu'il donne à l'histoire. Pour ces derniers, en effet, celle-ci a avant tout une fonction de légitimation du Code civil en ce qu'elle permet surtout de faire admettre la codification comme continuité au-delà de ses origines politiques, napoléoniennes, mais également et surtout révolutionnaires. Historiciser le droit est alors le moyen pratique de renouer les fils que Portalis avait déjà mis en exergue dès l'aube du siècle et que les juristes s'empressent de mettre en valeur qu'il s'agisse de promouvoir des origines romaines ${ }^{12}$ ou germaniques ${ }^{13} \mathrm{du}$ droit français ou de justifier telle ou telle institution. Sans même retenir le cas symptomatique mais excessif de Troplong ${ }^{14}$, l'histoire est alors souvent un véritable « réservoir d'opinions intemporelles sur les questions du Code ${ }^{15}$, une réserve d'arguments dans laquelle on puise le plus souvent à travers le filtre de la doctrine de Pothier et des auteurs modernes ; et si l'histoire du droit proprement dite est moins précieuse que le droit romain, son usage dogmatique est alors majoritaire pour asseoir prises de positions et dissertations ${ }^{16}$. Pour l'ami de Saleilles toutefois, cette œuvre d'acclimatation est belle et bien achevée et il n'a plus à y revenir. Si l'histoire doit jouer un quelconque rôle, il faut sans doute le chercher ailleurs et seul le contexte peut permettre de comprendre celui-ci. au projet scientifique et aux choix épistémologiques du doyen nan- 
céien. Il est vrai que ce dernier s'inscrit très largement et très logiquement dans le contexte de renouveau doctrinal de la Belle Époque ${ }^{17}$ profondément marquée tant par la défaite de Sedan, et donc par une crise «allemande » de l'Université française, que par la remise en cause du magistère civiliste et le développement de disciplines académiques concurrentes, ou encore par une République devenue conquérante, législatrice et laïque. Si l'on peut discuter de la participation de Gény à la construction du régime républicain ${ }^{18}$, il participe de toute évidence à un moment doctrinal et intellectuel fort, et pour le moins propice aux « cathédrales doctrinales » et aux grands systèmes. Reste toutefois qu'il ne faut pas sous-estimer, audelà de ces convergences et similarités certaines, notamment avec Saleilles et Hauriou ${ }^{19}$, les spécificités d'un projet scientifique qui ne peut être assimilé à ceux d'un Duguit ${ }^{20}$ ou d'un Lambert ${ }^{21}$, par exemple. Alors que ces derniers tentent de renouveler assez radicalement la méthode des juristes et repensent les fondements du droit, Gény propose davantage une adaptation de la méthode civiliste de son époque débarrassée toutefois de ses tensions positivistes du $\mathrm{xIX}^{\mathrm{e}}$ siècle ${ }^{22}$ et protégée des idées républicaines nouvelles. Bien informé des idées neuves, lecteur assidu de ses contemporains, notamment allemands ${ }^{23}$, il s'engage résolument et explicitement sur la voie d'un recentrement de la pensée juridique sur le travail du juriste et sur une dogmatique ancrée dans la tradition juridique. Alors qu'il revient sans cesse sur la prise en compte du donné social, ouvrant ainsi a priori la porte aux sciences sociales, le droit et la science qui l'accompagne demeurent bel et bien au cœur de son ambition de mettre au jour une science du droit immune et exclusive, et c'est finalement le seul «travail » des juristes qui le préoccupe tout au long de sa longue carrière ${ }^{24}$.

C'est donc pleinement en juriste que Gény s'attelle à l'immense tâche qui est la sienne. Il ne faut ainsi pas attendre de lui une œuvre philosophique ou même épistémologique, quel que soit l'affichage qu'il réalise lui-même. Son manque de rigueur, voire de profondeur, a été maintes fois souligné depuis Michel Villey ${ }^{25}$, et il ne faut donc nullement escompter quelques éblouissements intellectuels à la lecture d'un corpus volumineux et au style lourd et alambiqué. Il ne s'agit cependant nullement de passer son chemin mais de tenter, tout au contraire, de prendre au mot et à la lettre ce que le Nancéien nous dit 
de lui-même, mais également de ses contemporains qui le louent en majorité et lui ressemblent très souvent, à défaut de toujours le suivre, loin s'en faut.

7 Or, si l'on veut bien faire l'effort de scruter ses circonvolutions, les méandres de ses raisonnements, ses hésitations et ses multiples redites et retours en arrière, l'œuvre de Gény ne saurait être lue comme autre chose qu'un système idéologique. Lauteur ne s'en cache finalement nullement puisqu'il tourne sans cesse autour des mêmes questions qui sont toutes arrimées fermement à ses convictions personnelles que l'on peut qualifier sans hésiter de traditionalistes, à la fois conservatrices et catholiques ${ }^{26}$. L'une des caractéristiques de son œuvre est d'ailleurs la franchise que Saleilles ${ }^{27}$ met en exergue dans son introduction de 1899, et l'on ne saurait prétendre que Gény dissimule véritablement ses opinions ${ }^{28}$ d'ailleurs bien connues de tous ses contemporains. Si l'on accepte, à nouveau, de le lire attentivement, il les avoue même fréquemment alors même que son projet scientifique a pour objectif central de chasser le subjectivisme et l'idéalisme, et de bâtir une science juridique acceptable par tous et partout. Nulle supercherie donc, dans une œuvre qui tente pourtant de réaliser la prouesse de dépasser les antagonismes et les divergences pour sauver la « Maison du Droit » ${ }^{29}$ menacée et devenue fragile.

Si l'idéologie est une représentation du réel qui contient un projet d'agir sur celui-ci, alors l'œuvre juridique du doyen nancéien est bien idéologique. On objectera facilement qu'il ne faut pas oublier un contexte propice dans lequel le renouvellement des méthodes s'accompagne d'un renouveau des théories juridiques, des conceptions du droit et donc de prises de positions philosophiques et politiques. On relèvera également à raison que les fortes convictions personnelles d'un auteur, quelles qu'elles soient, ne peuvent tenir lieu de présomption irréfragable d'imprégnation idéologique de sa pensée et de son système. Reste que la science du droit qu'il nous propose est avant tout destinée à maintenir et contenir le droit dans ses cadres codifiés et établis de la fin du xix ${ }^{\mathrm{e}}$ siècle et du début du siècle suivant, c'est-à-dire à figer un modèle de société dans lequel le droit civil est la science sociale exclusive et devant demeurer entre les mains d'un groupe le plus homogène possible, groupe détenant de la sorte le magistère de parler au nom du « Droit ». En creux, mais explicitement, cela revient à promouvoir une science capable d'endiguer le flot 
croissant des transformations et revendications sociales, en somme, de toute promotion de la démocratie, car il s'agit bien là d'entendre la construction monolithique de Gény comme un rempart contre tout relativisme, toute transformation sociale, tout positivisme, et donc contre toute prise en charge exclusive du droit par un régime politique qui échapperait aux juristes considérés comme les gardiens de la vérité du «Droit ».

Quel peut alors être le rôle de l'histoire, simple science auxiliaire du droit, dans le projet scientifique de Gény ? Puisqu'il entend convaincre ses contemporains de la nécessité de le suivre dans la voie d'une science irréductible à toute autre et clairement fondée sur le droit naturel, il lui faut prendre position et statuer sur la place que les juristes doivent accorder ou concéder à l'histoire ou, plus exactement, à la méthode historique dans le raisonnement et l'épistémologie juridiques.

Or, deux difficultés conceptuelles majeures sont immanquablement rencontrées par le civiliste. D'une part, il lui faut concilier l'histoire et l'immuabilité du droit lui-même, ce qui pose derechef la question du droit naturel en ce que ce dernier est, pour lui, universel et immuable, alors que le droit dans l'histoire est par définition évolutif et contingent. Si le rapport au temps n'obsède certes pas le Nancéien, il lui faut tout de même penser cette articulation qui ne peut être esquivée totalement. Il lui reste alors, d'autre part, à intégrer au-delà du couple droit positif - droit naturel lui-même, le rapport de la société, voire de l'humanité à l'histoire puisque nul ne peut affirmer que le temps laisse totalement inchangé le monde et nier l'existence de multiples transformations du milieu politique, social ou économique. Passant de la question juridique à celle de linsertion du droit et du juriste dans un monde changeant, il se retrouve contraint de donner à son projet une dimension historique minimale, mais insusceptible de remettre en cause la science du droit qu'il entend promouvoir.

\section{Histoire et immuabilité du droit}

11 Afin de donner quelque intelligibilité au concept d'histoire dans l'œuvre de Gény, il convient de revenir sur sa compréhension du droit. Au premier abord, le civiliste a une conception du droit qui peut paraître dualiste en ce qu'il distingue le droit positif et le droit 
naturel. Il sépare donc a priori clairement l'un et l'autre droit, le second surplombant le premier qui serait le fait du seul législateur humain. C'est d'ailleurs ainsi qu'est le plus souvent comprise sa position relative à l'interprétation stricte de la loi, l'interprète ne pouvant prendre en compte que la volonté du législateur historique ${ }^{30}$, et son refus de toute coutume contra legem. Il ne cesse en effet de répéter qu'un texte législatif contraint le juriste au respect de sa lettre et que seule l'absence de sources contraignantes ouvre la porte à la libre recherche scientifique; et tel est bien le sens au moins apparent de son système de classement des sources du droit distinguant les sources formelles (loi et coutume) et les simples autorités (doctrine et jurisprudence). Il y aurait ainsi un droit positif issu des sources dites formelles et un droit naturel marginal bien qu'irréductible. Au sein du renouveau du droit naturel de la Belle Époque ${ }^{31}$, Gény est ainsi parfois classé parmi les tenants d'une forme de positivisme associé à une petite part de jusnaturalisme résiduel.

12 En réalité, ce dualisme dissimule très mal ce que l'auteur entend par droit positif et droit naturel, par positivité et nature. Pour lui, en effet, la loi écrite «ne peut être tenue pour autre chose, qu'une information, très limitée, du droit, résultant d'un ensemble d'injonctions, consacrées par un organe supérieur, à l'effet d'établir, sans conteste, quelques règles, qui ont paru susceptibles d'une formule nette, ou pratiquement indispensables ». De plus, et par voie de conséquence, «la décision légale tombe sous le coup du jugement rationnel, et, notamment [...] on la peut critiquer librement, en vue d'une réforme future ». La conclusion de son premier ouvrage de 1899 est limpide de ce point de vue :

Même sous sa forme positive, le droit nous apparaît comme un ensemble de règles, issues de la nature des choses, et devant être dégagées, au moyen d'une interprétation, plus ou moins libre, des éléments sociaux, qu'il a pour objet d'ordonner en vue du bien commun. Directement inspiré par la justice et l'utilité générale, son essence le place fort au-dessus des sources formelles, qui n'en sont que des révélations empiriques, destinées seulement à diriger les jugements humains de façon plus précise, mais, en soi, toujours incomplètes et imparfaites ${ }^{32}$. 
13 La notion de positivité est ainsi réécrite : est positive la nature des choses qui n'est autre que la réalité objective de ce qui est, et donc de l'être. Fait incontournable, cette réalité doit être appréhendée par le juriste qui fait œuvre de science véritable avant même de s'atteler à la « technique ». Il n'y a donc pas dans son système d'une part un droit naturel, et d'autre part un droit positif, mais bel et bien un droit positif issu des sources formelles qu'il s'agit d'interpréter strictement, et un droit positif inscrit dans les faits de nature qu'il convient de distinguer des simples faits de société, les premiers étant universels et immuables et les seconds variables, fragiles et fugaces, le droit naturel « positif »dominant bien entendu le droit du législateur ou la coutume. Les sources formelles du droit positif sont ainsi minorées et sa distinction du donné et du construit lui permet, à son sens, d'éviter tout subjectivisme car la science est connaissance objective du donné défini comme constat de «ce que révèle la « nature sociale », interprétée d'après elle-même ou suivant les inspirations d'un idéal supérieur, pour aboutir à des règles d'action, dont le fondement sera d'autant plus solide qu'elles contiendront moins d'artificiel ou d'arbitraire ${ }^{33}$. Si l'on n'inclut pas dans la positivité ce qu'il appelle la « nature des choses » et donc le droit naturel en tant que donné rationnel à côté des donnés réel ${ }^{34}$, historique ${ }^{35}$ et idéal ${ }^{36}$, toute sa construction s'effondre puisque il n'entend finalement parler que du « droit privé positif ». La structure même du corpus considérable qu'il construit sur une vingtaine d'années explicite et confirme cette analyse au-delà des divergences ou de la confusion de certains passages pris isolément. Son projet de chasser toute subjectivité et tout arbitraire de la science juridique, et donc de tendre vers l'objectivité de la science passe ainsi par une réinterprétation de notions et d'énoncés qui peuvent aisément tromper le lecteur.

14 Sa conclusion de Science et technique est pourtant pour le moins explicite :

Le « donné » correspond, à peu près, à la notion fondamentale de droit naturel. Il consiste en un fonds de vérités morales et économiques, qui, placées en présence des faits, commandent, pour les régir, certaines directions. Il se concentre autour de l'idée du « juste objectif », représentant un équilibre d'intérêts, qu'il faut parfois ausculter par toutes nos puissances pour connaître, mais qui ne fournit jamais qu'une orientation assez floue, de nature plutôt morale 
qu'économique, à vrai dire même, principalement morale. Ce « donné » reste la base essentielle du droit positif, mais ne peut avoir qu'une portée restreinte. Du moins, fournit-il la « règle », le «principe ${ }^{37}$.

Le but principal de la libre recherche scientifique est donc de découvrir « la nature des choses » qui demeure, malgré toutes les critiques dont elle fait l'objet à son époque, la notion « indispensable dans tout système de droit positif ». Pour lui, en effet, « la nature des choses, envisagée comme source (lato sensu) du droit positif, repose sur ce postulat, que les rapports de la vie sociale, ou, plus généralement, les éléments de fait de toute organisation juridique (du moins possible), portent en soi les conditions de leur équilibre, et découvrent, pour ainsi dire, eux-mêmes, la norme qui les doit régir ». L'effort scientifique n'est alors pas de se perdre en conjecture sur le but de cette nature ou sur la finalité de l'humanité, mais de découvrir « au-dessus des faits, la loi de leur harmonie ${ }^{38}$.

Ainsi donc, si le donné est un autre nom de la réalité, le droit naturel en forme le cœur et le fondement, et il ne saurait s'agir de n'y voir qu'une figure de rhétorique ou une maladresse de style car l'objet même de la science est donc de connaître la nature et le droit inscrit en elle. On aboutit ainsi à une confusion fondamentale du droit naturel et du droit positif, ce dernier n'étant nullement composé des seules règles posées par les autorités habilitées à ce faire, mais bien au contraire du droit naturel lui-même devant servir de critère «objectif »d'interprétation et de recherche de la solution positive ${ }^{39}$. Malgré quelques passages ambigus, il n'est donc plus une norme en surplomb et distincte, mais bel et bien un élément positif contraignant, et c'est bien là le sens profond de la positivité et de l'objectivité dont Gény nous parle sans cesse : est positive la règle contraignante pour le juriste en ce qu'elle conditionne et détermine la solution qu'il doit dégager en connaissant le donné et en mettant en ouvre celui-ci dans le cadre de la technique ${ }^{40}$. La loi est ainsi réduite à sa dimension technique la plus stricte et elle ne contraint l'interprète qu'en ce qu'elle s'impose, quand elle existe, comme point de départ obligatoire de son raisonnement et comme élément de l'analogie que Gény place au cœur de la méthode juridique ${ }^{41}$. Au surplus, la technique juridique elle-même finit par être assujettie au droit naturel puisque le Nancéien la définit non seulement comme la mise en œuvre pratique des 
résultats de la science et donc comme la formalisation de règles juridiques mais aussi comme «la réalisation effective de la justice entre les hommes ${ }^{42}$. Allant même plus loin, il affirme qu'il faut rechercher les éléments d'une «bonne technique juridique » dans « les exigences universelles [que] de la nature des choses nous indique $\gg^{43}$.

17 Aussi, admettre sans plus d'examen le positivisme, même partiel, de Gény relève du contresens si l'on veut bien ne pas jouer avec les mots et admettre que le positivisme implique non seulement le refus du droit naturel mais également, et surtout, l'acceptation en tant que droit de la règle simplement posée par l'autorité compétente ${ }^{44}$. Cette erreur ou ce malentendu profond a d'ailleurs très tôt été relevé par Georges Ripert ${ }^{45}$ dans son compte rendu de 1918 et le lecteur de Gény ne peut faire fi de ce que ce dernier déclare sans ambages au début de Science et technique :

\section{Il est clair, pourtant, que, n'étant que des modes contingents d'ex- pression d'une réalité permanente, les « sources » doivent rester su- bordonnées à cette réalité même. Et, comme un examen attentif du système cosmologique a dû ramener notre globe terrestre de son rang usurpé d'astre central à celui, plus modeste mais plus vrai, de planète solaire, ainsi une investigation mieux avisée des bases du droit placera la jurisprudence, la coutume, la loi elle-même, dans la dépendance de l'entité fondamentale, dont elles ne sont que des rayonnements partiels ${ }^{46}$.}

Très loin d'être une survivance ou une concession, le droit naturel occupe dès lors une place centrale dans le système du doyen de Nancy qui doit cependant faire face à la difficulté qu'il souligne très souvent lui-même, et qui n'est autre que le moyen de connaître ce droit. Mais posée en ces termes, la question finit par devenir l'objet même de toute sa construction car le «droit privé positif » se superpose au « Droit » lui-même en tant qu'il a un contenu nécessaire, ou du moins doit être articulé à une méthode conduisant à ce contenu.

Or, plusieurs questions préjudicielles interviennent ici. La première est celle des dangers du subjectivisme et de l'idéalisme que Saleilles met sans relâche en exergue ${ }^{47}$ et sur lesquels Gény revient très souvent. Pour ce dernier, il n'est en effet guère tenable d'affirmer que l'on peut déduire de quelques principes généraux du droit naturel un en- 
semble de règles objectives. Il affirme ainsi que l'idéalisme moderne ne peut plus convaincre tant ses excès sont patents et le conduisent à l'illusion rationaliste qui est pour lui une utopie pure et simple ${ }^{48}$. Il n'en demeure pas moins qu'en réalité, s'il chasse d'un côté toute connaissance a priori et subjective du droit naturel, critiquant fermement l'idéalisme moderne pour cela, il n'en inscrit que mieux le droit naturel dans le travail du juriste par le jeu de l'oscillation entre science et technique, et entre conscience et raison. Si en effet Gény n'a pas fait véritablement sienne la philosophie de Bergson ${ }^{49}$, il n'en tire pas moins la conclusion que la conscience est le prisme obligatoire d'une connaissance irréductible aux faits et au positivisme. Pour lui, connaissance scientifique et conscience doivent se compléter puisque la connaissance objective des faits ne peut que confirmer ce que la conscience ${ }^{50}$ nous dicte et ce que l'intuition nous suggère. Aussi peut-il écrire : «Il faut, pleins de confiance en notre conscience morale comme en notre raison, user de ces facultés, pour dégager les lois des phénomènes, et faire ainsi contribuer toutes nos ressources à une construction, vraiment scientifique, du droit commun que nous cherchons ${ }^{51}$. Il prescrit alors aux juristes, seuls titulaires d'une science sûre et ne pouvant réellement profiter des nouvelles sciences immatures et perfectibles que sont l'économie et la sociologie, de découvrir la justice et l'ordre, éléments centraux de la nature des choses qui simpose «dans le règlement de la vie juridique ». Il ne propose alors rien moins que de cumuler raison et conscience car ce que la première constate, la seconde l'impose puisque l'homme a une nature morale ${ }^{52}$ qui ne peut admettre que l'on sacrifie «le fonds, immuable et permanent, de vérité et de justice, qui s'impose à nous ». D’ailleurs, pour Gény, «malgré leur nihilisme doctrinaire [les plus fougueux champions des théories positivistes] reviennent, inconsciemment, à la reconnaissance effective d'un juste objectif, si réduite que puisse être sa portée ${ }^{53}$.

21 Puisque les choses ont une essence imparfaitement traduite dans les faits, « tout interprète du droit positif doit compter avec les inspirations de la raison et de la conscience, pour scruter le mystère du juste, avant de descendre à l'examen de cette nature des choses positives, qui précisera son diagnostic et mettra en œuvre les principes rationnels ». Et il poursuit : « Tout cela revient à dire, qu'il y a des principes de justice, supérieurs à la contingence des faits, et que, si 
les faits précisent la réalisation des principes, ils n'en sauraient contenir l'essence ${ }^{54}$. L'œuvre du juriste est donc de pleinement associer la raison, ou ce qu'il nomme l'intellectualisme, qui lui permet de connaître des faits, notamment grâce à l'analogie, et l'intuition confirmée par la croyance, sans toutefois oublier que la raison est faillible :

cette analogie n'est jamais une identité ontologique, et notre intuition des choses, qui, agissant à limage de l'instinct, ressent la plénitude de la vie sans pouvoir la pénétrer d'une façon pratique, nous avertit sans cesse de la duperie et des mensonges, au prix desquels les instruments de l'intellect, ne saisissant les réalités que par leurs côtés plastiques, et substituant des entités logiques aux choses réellement vivantes, nous permettent seulement d'entrer en contact avec le monde 55 .

Il ajoute un peu plus loin :

la science, quelque peu défaillante en ces matières, s'y trouve renforcée, sinon même remplacée, par cette révélation obscure, émanée des profondeurs les plus mystérieuses de l'âme, qu'on appelle la croyance, et qui, se rencontrant à la base de toute notre activité, semble, en même temps, indispensable et suffisante pour procurer les directions, sans lesquelles l'homme ne saurait assurer sa marche dans le sens de sa complète destinée ${ }^{56}$.

La vérité du Droit est de la sorte connaissable à qui veut bien user de sa conscience, utiliser ses croyances les plus intimes tout en recourant à une «saine logique juridique » telle qu'elle est décrite dans ses développements tant sur les sources du droit que sur la technique juridique. Vie et raison sont ainsi associées pour une pleine compréhension rationnelle et intuitive du Droit et révèlent une pensée dans laquelle le concept de science est bien peu précis ${ }^{57}$.

23 Si l'on peut être surpris par cette analyse qui part du rejet du subjectivisme et aboutit à combiner raison, conscience et croyances, l'étonnement ne cesse de croître, du moins à première vue, si l'on aborde la seconde question inévitable qui se pose à Gény et qui n'est autre que celle de la laïcité ou de la sécularisation du droit naturel en question. 
24 Au premier abord, Gény semble prendre le parti d'un droit naturel laïc. Parlant relativement peu du christianisme et de religion, malgré ses fortes convictions en la matière, il insiste au contraire sur la laïcité d'un droit qui n'est pas en lien direct avec les croyances, alors même qu'il fait de ces dernières un élément décisif par ailleurs. Inscrit dans la nature des choses, le droit naturel est donc présenté comme séparé de la religion, cette dernière n'ayant qu'un rôle très accessoire dans la science juridique. Il distingue ainsi le donné rationnel qui est le droit naturel et le donné idéal qui contient les considérations « d'ordre physique, psychologique, moral, religieux, économique, politique, qui, sans déterminer, de façon nécessitante, de nouveaux préceptes de conduite sociale, en projettent la direction à suivre ${ }^{58}$. Puisqu'il fait le constat de la diversité des religions au sein de l'humanité, il ne peut mettre en avant ce critère qui est, de plus et surtout, fort mal venu alors que la Troisième République affirme une laïcité de combat. Le christianisme devient ainsi un élément récurrent des donnés réels, historiques et idéaux, mais n'est plus le fondement affiché du donné rationnel qui est «le critérium décisif [qui] doit être recherché dans un domaine supérieur aux contingences et aux hasards de la vie ${ }^{59}$.

25 La stratégie du doyen nancéien est explicite : explorer la nature des choses permet de découvrir l'essence et la vérité première. C'est une question de science et de connaissance «objective », et non de «forces plus obscures, dont la valeur paraît discutable ${ }^{60}$. Or, pour convaincre et tenir son pari de bâtir une science véritable, il se trouve contraint de tout miser sur un droit naturel acceptable dans le contexte de la Belle Époque. Il insiste d'ailleurs sur le fait que la science porte pour l'essentiel sur celui-ci, seul point d'appui solide à l'activité rationnelle du juriste et à l'« activité personnelle et créatrice de l'esprit juridique ${ }^{61}$. Pour lui, « le droit naturel demeure le principe suprême de toute élaboration proprement scientifique du droit positif ${ }^{62}$.

26 Son entreprise de sauvegarde et de promotion de la tradition jusnaturaliste passe donc par un affichage qui ne tient pourtant pas longtemps puisque Gény cherche à convaincre tout en demeurant globalement honnête avec ses lecteurs qui connaissent fort bien ses engagements personnels et ses liens intimes avec la partie la plus conservatrice de l'Église catholique. S'il déclare en effet que le droit naturel 
qu'il défend est laïc car pour lui susceptible d'être accepté dans une société pluraliste, si à ses yeux le droit naturel est compatible avec la République, il ne peut cacher l'absence totale de sécularisation de son système :

Pour obtenir, de façon décisive, avec une plénitude assurée et une fermeté convaincante, le principe des normes directrices de l'activité morale et juridique, il faut avoir pris parti sur la conception générale de l'Univers, sur l'essence et la nature de l'homme, sur le mystère de son origine et de son existence en ce monde, sur l'énigme de sa destinée.

Il poursuit un peu plus loin en ces termes :

C'est donc, sur le simple fondement de l'expérience, interprétée à l'aide de toutes les puissances de la raison, que je veux affirmer ici le dualisme, voire le pluralisme, éclatant dans la constitution même de l'Univers, le théisme avec ses conséquences nécessaires, la distinction fondamentale entre l'homme et les autres êtres créés, l'immortalité de lâme humaine, sa liberté essentielle, sa sublime destinée vers Dieu ${ }^{63}$.

27 Pour lui, il faut ainsi refuser catégoriquement l'agnosticisme, le monisme matérialiste, le panthéisme et l'hypothèse évolutionniste car ces philosophies ignorent l'essence des choses, laissent dans une « obscurité désespérante » l'énigme du monde et aboutissent au scepticisme, à l'« indifférentisme philosophique » par refus de la métaphysique et de la transcendance ${ }^{64}$. Le projet de François Gény prend donc une toute autre physionomie qui ne peut être comprise autrement que comme une tentative de sauvetage d'un droit naturel chrétien au fondement finalement divin ${ }^{65}$ dans une société en voie de laïcisation. Il semble d'ailleurs que les lecteurs avertis de son œuvre ne puissent être dupes ${ }^{66}$ face à une dialectique peut-être essentiellement destinée aux non-initiés, même s'il est peu vraisemblable que le public étudiant soit visé. Peut-être s'agit-il plus simplement de fourbir des armes rhétoriques à tous ceux qui, parmi les juristes, partagent ses opinions et souhaitent éviter de s'enfermer dans une posture qui ne peut que subir les critiques venues à la fois du camp positiviste ou pseudo-positiviste, par exemple de Ripert, et des réformateurs, solidaristes ou jusnaturalistes mais libéraux ou « pro- 
gressistes », tels Duguit ou Lambert. Ainsi seule une lecture contextuelle prenant en compte le sous-texte et la prosopographie permet de comprendre une pensée qui ne peut être réduite à une sélection de citations isolées et aux légendes urbaines des facultés de droit et de ses concours.

Reste alors à dérouler les dernières conséquences de cette science finalement fondée sur la morale chrétienne ${ }^{67}$ puisque « la puissance de la vérité se transmet comme un fluide aux échelons qui suivent ${ }^{68}$. Pour Gény, le droit naturel ne peut être ainsi qu'universel et immuable si l'on veut bien ne pas s'attarder aux contingences de faits et aux vicissitudes des normes humaines :

Sans prétendre justifier, sous le rapport de la justice absolue, les institutions juridiques de toutes les époques et de tous les lieux, il suffit, pour écarter le scandale de leur diversité, d'observer que, presque toujours, le but a été moins différent que les moyens de le réaliser. Mais, si l'on admet, d'après la raison, l'expérience, et le sentiment intime, l'uniformité de la nature humaine, l'identité constante de sa destinée, et l'existence d'un ordre naturel permanent de rapports entre les éléments du monde, on en conclura nécessairement, que les principes de pure justice, qui ne sont qu'une des faces de cet ordre, conservent, au milieu des variétés et des contingences de leur mise en œuvre, un caractère universel et immuable ${ }^{69}$.

29 Si le « Droit » ou le droit naturel qui est au centre de son projet est universel et immuable, quelle place peut-il alors accorder à l'histoire? Pour résoudre cette équation, Gény part presque toujours des conclusions de l'« École historique » dont il reconnaît les mérites pour mieux en rejeter les conclusions. Il constate, en effet, «le triomphe » de cette école et souligne qu'elle a permis de dissiper les illusions du droit naturel moderne. Il note d'ailleurs que pour beaucoup de ses contemporains et prédécesseurs :

La vérité juridique ne saurait être une et identique à elle-même, que si les conditions sociales se rencontrent également identiques.

Conscients des nécessités contingentes de la vie du monde et pressentant les forces obscures, qui individualisent chaque fraction de l'humanité, les jurisconsultes, formés par la méthode historique, ont reconnu que l'idéal juridique prenait un aspect différent et se nuançait en mille teintes à travers les époques et les contrées diverses ${ }^{70}$. 
Si une petite part superficielle de relativité historique est acceptable, il ne saurait toutefois admettre de passer d'une illusion à une autre, et ainsi accepter un véritable relativisme. Il conclut donc nécessairement que « l'évolution du droit a un sens général, et, [...] en son fonds substantiel, elle reste placée au-dessus des contingences, de temps et d'espace ». Alors qu'il rejette souvent comme insoluble la question de la fin et du but, à chaque fois qu'il se penche sur la question de la relativité du droit, il est conduit à réintroduire la finalité, l'idée directrice et l'idéal que fournit justement le droit naturel qui devient ainsi à la fois un sein et un sollen ${ }^{71}$. Refusant tout évolutionnisme et tout relativisme fondamental, il est conduit à présenter le donné historique, et donc l'histoire, comme la succession des règles juridiques admises à diverses époques. Comme l'élément central du droit est permanent et inscrit dans une volonté divine, il est dès lors hors de question d'inscrire le droit dans la variété des faits historiques et dans le jeu des forces sociales contradictoires.

31 Gény commence donc toujours par admettre l'importance du donné historique :

\section{Le donné historique nous apparaît donc comme solidement établi et capable d'offrir à l'investigation scientifique du droit une base des plus sérieuses, bien que sujette au contrôle des facultés les plus hautes de l'esprit ${ }^{72}$.}

32 Il ne peut toutefois manquer d'aussitôt réduire la portée pratique de la connaissance historique en arguant notamment du fait qu'il n'est jamais possible de donner une direction sûre au droit à partir d'une expérience du passé toujours plus ou moins confuse, car seul le donné rationnel est à même de fixer «le critérium décisif » qui « doit être recherché dans un domaine supérieur aux contingences et aux hasards de la vie ». Il aboutit ainsi à réduire l'importance du donné historique qui n'est que «vague et universel dans ses grandes lignes, plus précis et particularisé dans ses détails ». Ce «droit préexistant » n'est pas négligeable et peut même avoir une autorité forte s'il se transforme en tradition, mais il reste sujet à caution et ne peut d'ailleurs nullement conforter les conclusions des sciences sociales et de la méthode déductive qui restent toujours des caricatures incapables d'atteindre la moindre parcelle de vérité ${ }^{73}$. 
33 Le droit devient ainsi anhistorique en ce qu'il est doté d'une essence universelle et immuable, et l'on comprend mieux l'hostilité fondamentale de Gény pour le droit naturel à contenu variable de Stammler et contre toute forme de relativité historique. Défenseur d'un droit naturel très classique jusque dans l'entre-deux-guerres et audelà, le doyen de Nancy entend refonder la science juridique sur un socle inaltérable, sur une vérité une et indivisible. L'histoire vient donc ici buter contre la structure même du projet qu'il défend et ne peut plus recevoir qu'un rôle très marginal. Le Nancéien ne peut donc faire du monde social qu'un théâtre d'ombres dans lequel seule l'expérience de la vérité des choses peut être recherchée et guider le juriste. Si la science n'a pour but que de découvrir cet ordre, les circonstances historiques ne peuvent être que les épreuves successives d'une vérité atemporelle, la répétition d'actes et d'idées issus d'une même vérité que les historiens peinent à voir s'ils ne sont pas euxmêmes éclairés par la connaissance de la nature des choses.

Gény oscille ainsi sans cesse entre les divers impératifs de son système pour trouver le chemin de la tradition. Comme il est contraint par la constitution politique de reconnaître à la loi un rôle minimal ${ }^{74}$ et comme le Code civil s'impose à lui comme pierre angulaire du droit, il admet que le droit positif, au sens strict, joue un rôle central dans la technique juridique. Il libère ainsi toute l'œuvre scientifique du juriste qui peut être consacrée à la connaissance du donné ou autrement dit du droit naturel. Mais admettre cela le conduit inévitablement à étoffer et à étendre le domaine d'observation du juriste à tout ce que la réalité peut révéler. Retrouvant en l'occurrence la dichotomie classique entre forme et fond, entre matière et esprit, entre donné et construit, il tente de convaincre son lecteur que tout cela converge inévitablement vers quelques vérités premières et donc atemporelles. Chemin faisant, il ne peut donc qu'abandonner ou restreindre tout ce qui est susceptible de perturber la «bonne lecture » de la réalité et l'histoire ne peut manquer de poser d'importantes difficultés.

35 Le «Droit » est en quelque sorte fixé en un point anhistorique puisque sa conception de la nature, ne pouvant dissoudre toute trame historique, rend caduque toute possibilité de contradiction et toute variation véritable, et l'histoire ne peut être qu'une succession de faits dont la signification véritable appartient au juriste. Autrement 
dit, l'histoire ou plus précisément l'argument historique n'est recevable que s'il est une épreuve utile d'une même vérité. Les faits historiques peuvent errer, montrer les déviances des uns et les bonnes actions des autres, mais jamais montrer la moindre relativité profonde car ce serait nier l'existence même du droit naturel universel et immuable, du moins si l'on ne considère que la tradition juridique occidentale. L'histoire devient une trame temporelle où viennent s'inscrire des scènes dépourvues de réalités et de sens propre, ou autrement dit de toute spécificité. Elle est ainsi une succession d'actes que l'on peut appréhender exactement comme le droit comparé, en terme de modèle, et ressemble fort à une expérience humaine toujours recommencée, ici ou là, hier comme aujourd'hui, et ce depuis la philosophie grecque au moins.

Histoire et droit sont donc avant tout des interprétations bonnes ou mauvaises, et seule compte in fine la lecture qui vise et tombe juste puisqu'elle révèle ce qui a été révélé. En sa qualité de juriste, et compte tenu de ses conceptions en la matière, Gény ne peut donc donner à l'histoire qu'un rôle très subsidiaire, purement dogmatique et qui ne peut être autre que de montrer l'expérience vécue dans le temps du « Droit ».

\section{Histoire et évolutions de la so- ciété}

37 Gény ne se contente pas de quelques déclarations de principe sur l'utilité de la méthode historique et consacre quelques passages intéressants à la place de l'histoire dans la méthode juridique. Puisqu'il ne peut nier les changements historiques les plus évidents ${ }^{75}$ et comme il a pleinement conscience que l'histoire est une arme qu'il faut utiliser à bon escient, il prend un soin particulier à souligner les dangers d'une mauvaise interprétation des faits. En cela il semble avoir compris l'apport de Savigny tout en conciliant son fixisme jusnaturaliste avec une évolution acceptable.

38 L'histoire du droit ne saurait en effet montrer la succession des faits bruts en ce qu'ils seraient susceptibles de mettre au jour les aspirations, les luttes, les rapports de force et donc les choix, les consensus ou les diverses formes de domination dont le droit serait le reflet ou 
le résultat. En d'autres termes, bien que lecteur de Jhering ${ }^{76}$, Gény ne peut admettre le rôle des acteurs dans l'histoire et met en garde ses contemporains contre le chaos des faits historiques. Au même titre que l'opinion, cette « grande trompeuse » dont il faut percer les « obscurités et les mensonges " ${ }^{77}$, les faits historiques restent éminemment suspects. Loin de permettre une ouverture aux sciences sociales $^{78}$, son système bannit toute prise en compte directe de la connaissance historique ${ }^{79}$.

39 Dans toute son œuvre, et donc de façon récurrente, il insiste sur le 《mystère ${ }^{80}$ qui entoure l'élaboration du droit, ce « jeu combiné de forces sociales obscures ${ }^{81}$ qu'il oppose toujours à l'évidence de la science et donc du droit naturel qui s'impose à nos consciences :

Ici, en effet, nous pénétrons sur un domaine rationnel, où l'observation et l'histoire ne sont plus que des guides initiaux, où l'idéal ne peut encore que faire entrevoir une expansion ultérieure, où seule la conscience, appuyée par les force d'intelligibilité et de logique, est capable de fournir une lumière féconde et d'offrir une direction sûre ${ }^{82}$.

À l'exclusion du sociologue ou de l'économiste, mais également de l'historien, seul le juriste peut donc faire la lumière et séparer le bon grain de l'ivraie, car « ce sera notre effort propre, de dégager, dans la masse, un peu chaotique, du monde social, les éléments, vraiment positifs, doués de la fermeté suffisante, pour fournir les solutions nécessaires ${ }^{83}$. Prétextant le besoin de sécurité juridique, ce grand totem de la pensée juridique, il entend ne jamais admettre l'information historique autrement qu'assujettie à une interprétation jusnaturaliste :

Ici interviendra l'histoire, et, plus précisément, toute la tradition de notre droit privé, pour nous instruire par l'expérience du passé, et mettre au point le moment exact de civilisation, qui doit rester seul décisif. Et, sans doute, la révélation, souvent confuse par sa complexité même, toujours mouvante et imparfaite dans sa signification précise, qui résultera d'un semblable examen, n'aura jamais, ni la rigueur de détermination, ni la force de conviction qui s'attachent aux sources formelles. Il n'en reste pas moins vrai, qu'elle nous présente, à l'image et un peu au dessous de l'analogie proprement dite, un procédé de découverte juridique, tirant sa force d'une nature des choses 
vraiment positive, et procurant à l'interprète des éléments objectifs d'appréciation, capables d'assurer une sérieuse direction à son jugement ${ }^{84}$.

41 Puisqu'il faut « arracher le secret des règles » du droit, et bien qu'il affirme que l'activité du juriste se fait sous le contrôle précieux des « résultats de l'expérience humaine, vivifiant et fécondant souvent la réflexion », puisque « tout se ramène à l'histoire, la grande maîtresse d'expérience en matière sociale ${ }^{85}$, l'essentiel demeure l'analyse juridique car «seules, en effet, peuvent convenir à ce but des disciplines, de tendance normative, qui, en même temps qu'elles analysent les faits de la vie sociale, suggèrent la direction, suivant laquelle s'établit l'ordre nécessaire pour les régir ${ }^{86}$.

42 Aussi faut-il éviter scrupuleusement la « séduction, trop aisément absorbante, de la pure érudition » et soumettre l'histoire à un contrôle constant $^{87}$. Si cette dernière a pu procurer un «soutien solide » quand «mettant en œuvre avec des visées nettement pratiques, les résultats obtenus par des procédés d'investigation sévèrement contrôlés, elle s'efforce à dégager, de l'exemple des précédents, la direction la mieux adaptée aux évolutions de la vie sociale contemporaine ${ }^{88}$, il observe un déclin de cet apport de l'histoire au droit au début du xx ${ }^{\mathrm{e}}$ siècle.

43 Il récuse, en l'occurrence, l'apport de la méthode historique de Saleilles qui n'est pas parvenu «à nous indiquer exactement, par quels moyens l'histoire, ainsi comprise, arriverait à régénérer effectivement l'interprétation moderne » ${ }^{89}$. Pour lui, au contraire, l'histoire n'est pas un « instrument efficace et sûr » car les solutions du passé ne se transforment pas en coutume, et surtout parce que la « seule considération du passé reste impuissante à supplanter » les critères de valeur objective : l'histoire est défaillante car « elle ne fournit pas, en elle-même et à elle seule, les critères de validité des règles ». Allant plus loin, il affirme que «l'idée, qui fait la valeur de l'histoire du droit (idée d'évolution), condamne, chez elle, toute prétention, à fournir des règles, pleines et sûres, à la vie ${ }^{90}$. Ainsi seule la tradition définie comme l'« ensemble des solutions juridiques transmises à une époque par les époques précédentes » ${ }^{91}$ peut constituer un élément fiable dans l'activité du juriste et donc une histoire acceptable. 
44 C'est alors qu'apparaissent les ressources de l'histoire qui se résument pour Gény à deux éléments : autorité et tradition. La pensée de Gény sur ce point est assez convenue mais recèle de précieuses indications sur le rôle donné à l'histoire par de nombreux juristes. Il commence en effet par lier tradition et autorité. Pour lui, la tradition ne saurait être confondue avec la coutume puisqu'elle ne repose sur aucune « conviction commune ». Elle est une simple autorité « revêtue d'un cachet d'antiquité, qui lui donne, à la fois, le prestige et la caducité d'un grand âge » ${ }^{92}$, un «pur élément historique ». Toutefois, contrairement à l'ancien droit prérévolutionnaire au sein duquel autorité et tradition jouaient un rôle similaire à celui du droit romain, c'est-à-dire le rôle d'une raison écrite avec comme élément clef la communis opinio doctorum, la tradition est pour lui composée des autorités anciennes qui ne peuvent s'imposer à son époque. La codification a en effet rompu la « chaîne des temps » car elle fournit «à la culture juridique, un sol, sinon vierge, du moins défoncé à nouveau, et purgé de tout le fouillis inextricable de ronces et d'épines, dont les siècles l'avaient couvert ». Classiquement, il distingue les matières codifiées au sein desquelles « les traditions, antérieures à chacune de ces codifications, ne conservent pas aujourd'hui la force créatrice, qu'elles avaient autrefois », et les matières non codifiées pour lesquelles « les autorités anciennes doivent conserver leur force [...] atténuée seulement par l'effet du temps et par la réaction inévitable de tout le mouvement codificateur sur l'ensemble du droit privé » ${ }^{93}$.

45 Est-ce à dire qu'est ainsi détruite « la part de l'élément historique dans l'élaboration d'ensemble de notre droit positif »? Sur ce point sa position semble claire :

Il ne s'agit pas, suivant une pratique encore trop répandue, de puiser, dans tout le fonds ancien, des passages quelconques, ramassés un peu au hasard de la découverte, comme des armes d'occasion, à l'appui d'une thèse préférée [...] on doit dire, que, sur les points compris dans le domaine des codifications, et qu'il n'a pas positivement réglés, le législateur a nécessairement laissé plus grande liberté d'appréciation à l'interprète. $\mathrm{Si}$, donc, celui-ci a recours, pour éclairer son jugement, aux traditions anciennes, il ne les prend, qu'en ellesmêmes, à titre de simples autorités. Or, je n'aperçois vraiment pas, comment, ainsi envisagées, ces traditions pourraient avoir survécu à la table rase de la codification, avec la force qu'elles avaient autrefois, 
alors qu'elles étaient dans leur milieu d'éclosion, aujourd'hui complétement transformé, et qu'elles s'harmonisaient avec un système juridique, qui se trouve, à l'heure actuelle, gravement altéré par les Codes ${ }^{94}$.

Aussi apparaît-il a priori pleinement conscient des limites d'une histoire dogmatique telle que pratiquée par ses prédécesseurs et contemporains, et il ne saurait admettre sans se contredire que les textes juridiques du passé constituent des vérités juridiques. Dans un même mouvement, il évite également toute accusation de traditionalisme en déniant à la tradition le caractère d'élément décisif et contraignant au sein de sa libre recherche scientifique.

47 Les choses sont pourtant moins nettes lorsqu'il revient sur la question de la valeur de ces traditions dans le cadre du travail du juriste. Car, si pour lui les traditions ne sont pas des sources formelles, « elles n'en conservent pas moins, très justement, sur l'esprit du juriste, un ascendant moral, que rien ne saurait leur ravir. Par-dessus tout, elles gardent toute la valeur pratique de l'histoire, je veux dire, qu'elles servent d'enseignement, permettant de juger, d'après l'expérience de la vie juridique, le mérite social et la valeur de fond des institutions $\gg 95$.

48 Gény éclaire sa position lorsqu'il passe de l'analyse de la tradition à celle de l'autorité car « entre Tradition et Autorité proprement dite (moderne), il n'existe pas, sauf circonstances particulières, une différence de nature, plutôt seulement une différence d'époque et de durée ${ }^{96}$. Pour lui en effet, les autorités sont les traditions du temps présent, et il confesse sa fidélité à l'autorité tout en ne pouvant l'admettre comme source formelle, au même titre que la tradition d'ailleurs. Sa citation de Balfour est éclairante :

\footnotetext{
Nous n'oublierons pas que c'est à l'autorité plutôt qu'à la raison que nous devons non seulement la religion, mais la morale et la politique ; que c'est l'autorité qui nous a fourni les éléments essentiels des prémisses de la science ; que c'est l'autorité plus que la raison qui a solidement jeté les bases de la vie sociale ; que c'est l'autorité plus que la raison qui a cimenté l'édifice.
}

Il poursuit de façon non moins explicite : 
dans les recherches, qui ont l'homme pour objet, et où sa volonté joue un rôle essentiel, le sentiment de la vérité ne se fait jour que par l'exercice même de cette volonté, et ne s'affirme que par une accumulation d'exemples et de précédents, où chaque époque entre pour sa part [...] Ayons le courage de confesser notre fidélité à la tradition, notre attachement à l'autorité 97 .

49 Il invite alors les juristes à s'atteler à la recherche de l'« intensité de clarté objective »de ces éléments indispensables que sont l'autorité et la tradition ${ }^{98}$. S'il est bien difficile de comprendre une telle proposition, il n'en demeure pas moins qu'il intègre pleinement tant l'autorité que la tradition dans l'œuvre propre de la science et de la technique. Ne deviennent ainsi acceptables que ce qui est qualifiable de tradition ou d'autorité, autrement dit ce qui passe par le filtre de la communauté des juristes qui a le pouvoir de vérifier la recevabilité des idées et des règles. De l'histoire ne reste ainsi que ce qu'il nomme tradition ou ce que l'on pourrait définir comme le «langage définitoire et classificatoire du passé » rejoué dans le présent ${ }^{99}$. Les faits sont presque tous disqualifiés puisqu'ils sont par trop chaotiques et incertains. Conformément à ses convictions jusnaturalistes, Gény ne peut donc conserver que les épreuves de la vérité intangible, et les traditions admises par la mémoire des juristes.

50 L'histoire est ainsi réduite à la mémoire, aux traditions inventées et acceptées par le groupe au même titre que les autorités sont celles qui sont définies comme telles par les juristes. En témoigne d'ailleurs le lien que Gény instaure entre histoire et valeurs de la civilisation ${ }^{100}$. Seule finalement une histoire des textes et des idées devient acceptable, parce que mobilisable, au sein d'une méthode articulée à un pivot central invariable. Il s'agit donc d'une histoire pleinement axiologique, où la tradition est la simple expérience du passé revue et corrigée à chaque génération de juristes. Elle doit répondre aux enjeux du présent et servir d'arguments dans le jeu doctrinal d'interprétation du droit positif.

51 Gény ne propose donc pas autre chose en matière d'histoire que ses prédécesseurs civilistes du $\mathrm{XIX}^{\mathrm{e}}$ siècle. La science auxiliaire du droit dont il parle est bel et bien aussi dogmatique et axiologique que précédemment, et seule la justification et la naturalisation du Code civil ont disparu des préoccupations du $\mathrm{xx}^{\mathrm{e}}$ siècle. En quelque sorte, Gény 
actualise une pratique de l'histoire qui reste pleinement instrumentale et doctrinale. Ce sont toujours les membres de la doctrine juridique qui doivent procéder à linterprétation idoine qui seule donne quelque valeur normative aux faits ou aux textes du passé. La normativité est un monopole du juriste qui sait ainsi déceler le droit au-delà des faits et des évènements. Plus encore, cette histoire est soumise au contrôle de la conscience et de l'intuition du même juriste qui détient la clef de répartition du droit et du non-droit, de l'ordre et du chaos.

52 Cette « histoire axiologique », qui peut être définie comme l" « histoire des œuvres qui ont mérité de demeurer, traitées comme vivantes, éternelles, non comme relatives à leur temps » ${ }^{101}$ est permise en ce qu'elle est inscription temporelle et recherche de la singularité qui est ici une autre façon de penser l'exemplarité d'une expérience historique de la vérité. Cette histoire est ainsi celle « des vérités établies et de leur acquisition » ${ }^{102}$, car tout n'est pas évidemment donné et l'évolution opère comme le temps qui s'égrène et ne peut se répéter à l'identique. Aussi Gény reconnait-il une certaine forme d'historicité tout en ne pouvant admettre qu'une histoire officielle avec ses grands hommes, ses grandes idées et donc ses vérités que l'on peut rechercher dans le mimétisme le plus sûr, dans ce qui fait autorité, dans ce que la tradition a conservé, autrement dit ce qui mérite d'être conservé. L'histoire qu'il peut admettre est celle qui démontre ${ }^{103}$ et légitime le droit tel qu'il est, parfois, et le plus souvent tel qu'il devrait être ou tel qu'il a été parce qu'il devait en être ainsi. Il ne saurait toutefois s'agir d'une « histoire pure » qui devrait s'attacher au spécifique, au désordre des situations et aux diverses et variables « opérations du droit » ou « technologies juridiques » ${ }^{104}$. Or, c'est ce que ne peut accepter Gény qui refuse justement ce chaos contraire à l'ontologie et à l'axiologie qui sont les siennes et celles de son groupe d'ailleurs. Son hostilité à la démocratie rejoint ici pleinement sa méfiance envers l'histoire par peur de cette même vie chaotique et tumultueuse, et par choix politique de la filiation et de l'immutabilité. Si la démocratie est autonomie du sujet ${ }^{105}$, Gény nous plonge dans un monde hétéronome qui fixe en un point axial la nature véritable des choses et des êtres.

53 Si autorité et raison sont synonymes, si l'interprétation est l'œuvre propre du juriste, si la vérité est connaissable et connue, l'histoire ne 
peut fournir que l'intrigue ou le scénario que suggère François Gény qui, à rebours des titres de ses ouvrages et de son ambition, ne peut que nous proposer un «sommeil dogmatique ». Il semble en cela illustrer une tendance profonde des juristes, voire des historiens du droit eux-mêmes ${ }^{106}$, à éviter ou même à nier l'inscription du droit dans la société, dans une histoire intellectuelle, pour ne parler que de celle-ci, qui ne peut se limiter, sans appauvrissement regrettable, à l'étude de la succession des œuvres produites par les juristes par d'autres juristes qui réinventent leurs traditions et leurs grands ancêtres pour mieux légitimer leur pouvoir. Si l'histoire est l'écriture ou la reconstruction objective de la spécificité de tous les faits irrémédiablement perdus dans l'épaisseur du temps, Gény ne nous propose finalement qu'une histoire immobile qu'il faut pour le moins tenter de comprendre pour ce qu'elle est, sans à avoir à statuer sur la figure qu'il devrait, ou non, incarner dans la mémoire collective des juristes.

\section{NOTES}

1 Parmi l'abondante bibliographie, on retiendra spécialement E. Gaudemet, L'interprétation du Code civil en France depuis 1804 (1935), Paris, 2002, p. 120-130; Recueil d'études sur les sources du droit en l'honneur de François Gény, Paris, 1934, 2 vol. ; M. Toutsakevitch, Élaboration scientifique du droit positif dans la conception de François Gény, thèse droit, Paris, 1938 ; Fr. Terré, «En relisant Gény », Archives de philosophie du droit, 6, 1961, p. 125-140 ; Le centenaire du doyen François Gény. Recueil des conférences prononcées les 26 et 27 octobre 1962, Paris, 1963 ; O. Cayla, «Lindicible droit naturel de François Gény », Revue d'histoire des facultés de droit et de la science juridique, 6, 1988, p. 103-122 ; «François Gény e la scienza giuridica del novencento », Quaderni fiorentini per la storia del pensiero giuridico moderno, 20, 1991, p. 1-540 ; Cl. Thomasset, J. Vanderlinden et Ph. Jestaz (dir.), François Gény, mythe et réalités 1899-1999. Centenaire de Méthode d'interprétation et sources en droit privé positif. Essai critique, Montréal Paris, 2000 ; Ph. Jestaz et Chr. Jamin, La doctrine, Paris, Dalloz, 2004, spécialement p. 133-165 ; N. Dissaux, «Linfluence de Bergson sur les idées du doyen Gény », Revue trimestrielle de droit civil, 3, 2008, p. 417-429 ; J. Boulaire, "François Gény et le législateur », dans Le renouveau de la doctrine française. Les grands auteurs de la pensée juridique au tournant $d u \mathrm{xx}^{e}$ siècle, N. Hakim et F. Melleray (dir.), Paris, Dalloz, 2009, p. 69-94 ; M. Auberger, 
«Un autre regard sur François Gény et son œuvre », Revue trimestrielle de droit civil, 2, 2010, p. 261-279 ; C. Thomasset, «Épilogue : l'actualité de François Gény et de son œuvre », Revue trimestrielle de droit civil, 2, 2010, p. 279-284 ; J.-P. Chazal, « Léon Duguit et François Gény, controverse sur la rénovation de la science juridique », Revue interdisciplinaire d'études juridiques, 2010/2, 65, p. 85-133 ; O. Cachard, Fr. X. Licari et Fr. Lormant, La pensée de François Gény, Paris, Dalloz, 2013. Sur ce dernier ouvrage, cf. N. Hakim, « Olivier Cachard, François-Xavier Licari et François Lormant (dir.), La pensée de François Gény, Dalloz, 2013 », Revue trimestrielle de droit civil, 2, 2013,p. 467-470. Cf. également C. Jamin, F. Audren et S. Bloquet, Correspondance Saleilles Gény, Paris, Lextenso, à paraître en 2016.

2 Méthode d'interprétation et sources en droit privé positif. Essai critique, $2^{\mathrm{e}}$ éd. revue et mise au courant, Paris, LGDJ, 1919, 2 tomes.

3 Science et technique en droit privé positif, Nouvelle contribution à la critique de la méthode juridique, I, Introduction, Première partie - Position actuelle du problème du droit positif et éléments de sa solution, Paris, Sirey, 1914 ; II, Seconde partie - Élaboration scientifique du droit positif (L'irréductible droit naturel), Paris, Sirey, 1915 ; III, Troisième partie - Élaboration technique du droit positif, Paris, Sirey, 1922 ; IV, Quatrième et dernière partie Rapports entre l'élaboration scientifique et l'élaboration technique du droit positif (Le conflit du droit naturel et de la loi positive), Paris, Sirey, 1924.

4 Cf. toutefois les remarques de Christophe Jamin qui relève que Gény règle ses comptes avec Savigny dès 1899 et remplace l'histoire dans ses fonctions légitimatrices par la libre recherche scientifique («François Gény d'un siècle à l'autre », François Gény, mythe et réalités, op. cit., p. 15-17). Cf. également N. Pierre, «François Gény et la responsabilité civile : le droitscience et le sens de l'histoire », La pensée de François Gény, op. cit., p. 153171 et B. Frydman, «Le projet scientifique de François Gény », François Gény, mythe et réalités, op. cit., p. 214.

5 Cf. spécialement «Quelques mots sur le rôle de la méthode historique dans l'enseignement du droit », Revue internationale de l'enseignement, 19, 1890, p. 482-496 et «Le Code civil et la Méthode historique », Le Code civil, 1804-1904. Livre du centenaire publié par la Société d'études législatives, Paris, 1904, tome 1, p. 97-129. Sur Saleilles, cf. récemment F. Audren, C. Chêne, N. Mathey et A. Vergne (dir.), Raymond Saleilles et au-delà, Paris, Dalloz, 2013 ainsi que C. M. Herrera, Y a-t-il vraiment une crise de la science politique ?, Paris, Dalloz, 2012 et M. Xifaras, « La veritas iuris selon Raymond Saleilles. Remarques sur un projet de restauration du juridisme », Droits, 
Revue française de théorie, de philosophie et de culture juridiques, 47, 2008, p. $77-148$.

6 Méthode d'interprétation, op. cit., tome 2, 1919, nº 143.

7 Sur celui-ci, cf. P. Jestaz et C. Jamin, « En relisant Eugène Gaudemet », présentation de la réédition de E. Gaudemet, L'interprétation du Code civil en France depuis 1804 (1935), Paris, La Mémoire du Droit, 2002, p. 9-36 ; C. Pérès, «Eugène Gaudemet : l'historien des doctrines », Hommage à Eugène Gaudemet, Paris, Dalloz, 2015, p. 49-67.

8 Sur Bonnecase, cf. C. Jamin, « Julien Bonnecase et Charles Demolombe », Revue trimestrielle de droit civil, 1992, p. 856-857 ; N. Hakim, « Julien Bonnecase : historien de la science juridique ? », Histoire de l'histoire du droit, J. Poumarède (dir.), Études d'histoire du droit et des idées politiques, 10, 2006, Toulouse, p. 291-302 ; F. Cherfouh, Le juriste entre science et politique : la Revue générale du droit, de la législation et de la jurisprudence en France et à l'étranger (1877-1938), thèse droit, Bordeaux IV, dactyl., 2010 ; N. Hakim, «Socialisation du droit et romantisme juridique : autour d'une controverse entre Julien Bonnecase et Paul Cuche », De la terre à l'usine : des hommes et du droit. Mélanges offerts à Gérard Aubin, B. Gallinato-Contino et N. Hakim (dir.), Bordeaux, Presses universitaires de Bordeaux, 2014, p. 139-173.

9 Notons qu'en réalité la rupture avec l'exégèse est sans doute plus superficielle que réelle ne serait-ce que parce que Gény reste imprégné du dogme du respect de la volonté du législateur historique et donc d'une lecture de type exégétique du Code civil. En ce sens, cf. not. B. Frydman, op. cit., p. 218-219.

10 Son chapitre intitulé « Évolution de la méthode juridique 》 ne fait que 7 pages et il ne s'encombre d'aucune perspective historique (Méthode d'interprétation, op. cit., tome $\left.1, \mathrm{n}^{\mathrm{os}} 8-11\right)$. L'essentiel du tome premier consacré à l'exposé analytique de la méthode traditionnelle puis à la critique négative de cette méthode est, de ce point de vue, tout à fait similaire. Il faudra attendre 1950 pour voir Gény inscrire ses analyses dans une chronologie de la pensée juridique alors que, justement, Gaudemet et Bonnecase ont déjà accompli ce travail («L'évolution contemporaine de la pensée juridique dans la doctrine française », Le droit privé français au milieu $d u \mathrm{xx}^{e}$ siècle. Études offertes à Georges Ripert, Paris, LGDJ, 1950, tome 1, p. 3-8).

11 Le cas de Planiol est ici éclairant, même s'il faut aussitôt préciser que Gény produit une œuvre de nature et de forme toute différentes. Sur Planiol, cf. P. Rémy, « Planiol : un civiliste à la Belle Époque », Revue trimestrielle 
de droit civil, 2002, 1, p. 31-45 ; G. Babert,Le système de Planiol (Bilan d'un moment doctrinal), thèse droit, Poitiers, dactyl., 2002.

12 Nous pouvons citer ici spécialement le cas de Troplong, ardent défenseur des origines romaines ou encore de Claude Bufnoir. D'autres, en revanche, sont plus réservés à l'instar de Charles Demolombe qui n'admet cette filiation que sous bénéfice d'inventaire.

13 L'exemple le plus évident est en l'occurrence François Laurent selon lequel il faut accorder une place de premier ordre à l'histoire et reléguer le droit romain à un rôle secondaire dans les sources du Code civil (cf. par exemple ses Principes de droit civil, Bruylant et A. Durand \& L. Pédone, 1869, tome $\left.1^{\mathrm{er}}, \mathrm{n}^{\mathrm{o}} 24, \mathrm{p} .36\right)$. Il écrit notamment : «Cela devait être, et il est heureux que cela soit. Le droit est un élément de la vie des peuples, il se modifie donc avec la vie. Nos sentiments, nos idées, notre civilisation ne sont plus ce qu'ils étaient à Rome ; nous ne sommes plus des Romains, comment notre droit serait-il encore celui d'un peuple dont nous différons sous tant de rapports ? Sans doute, la civilisation romaine est un des éléments de la civilisation moderne, et le droit y joue le grand rôle ; mais ce n'est pas l'élément dominant » (Ibidem, no 23, p. 32-33). Il ajoute plus loin : « Les Romains, peuple de juristes, ont la gloire d'avoir porté la science du droit à la perfection, mais ils ont payé cher cette gloire [...] C'est une race formaliste, dure, impérieuse, sans cœur, toute de calcul » (ibid., no 24, p. 33 ; il cite ici son ouvrage Études sur l'histoire de l'humanité, $2^{\mathrm{e}}$ éd., p. 125). Sur Laurent, cf. J. Erauw, Liber memorialis François Laurent 1810-1887, Bruxelles, StoryScientia, 1989.

14 Sur Troplong, cf. P. Rémy, « Préface aux préfaces », Revue d'histoire des Facultés de droit et de la science juridique, 18, 1997, p. 161-188 ; M. Xifaras, «L'école de l'exégèse était-elle historique ? Le cas de Raymond-Théodore Troplong (1795-1869), lecteur de Friedrich Carl von Savigny », Influences et réceptions mutuelles du droit et de la philosophie en France et en Allemagne, J.-F. Kervégan et H. Mohnhaupt (dir.), Francfort-sur-le-Main, 2001, p. 177209.

15 P. Rémy, « Préface », op. cit., nº 15, p. 182.

16 Sur cette question entendue largement, cf. F. Cherfouh, « Les usages de l'histoire et du droit romain dans les ouvrages d'Introduction au droit », Histoire des manuels de droit. Une histoire de la littérature juridique comme forme $d u$ discours universitaire, A.-S. Chambost (dir.), Paris, LGDJ, 2014, p. 95-110. 
17 Sur ce moment doctrinal décisif, cf. spécialement C. Jamin, « L'oubli et la science. Regard partiel sur l'évolution de la doctrine positiviste à la charnière des $\mathrm{XIX}^{\mathrm{e}}$ et $\mathrm{xx}^{\mathrm{e}}$ siècles », Revue trimestrielle de droit civil, 1994, p. 815827 ; du même, «Dix-neuf cent : Crise et renouveau dans la culture juridique », Dictionnaire de la culture juridique, D. Alland et S. Rials (dir.), Paris, Lamy-PUF, 2003, p. 380 et La cuisine du droit. L'École de Droit de Sciences Po : une expérimentation française, Paris, Lextenso, 2012 ; P. Jestaz et C. Jamin, La doctrine, Dalloz, 2004, spécialement p. 95 et s. ; C. Jamin et P.-Y. Verkindt, «Droit civil et droit social : l'invention du style néoclassique chez les juristes français au début du $\mathrm{xx}^{\mathrm{e}}$ siècle », Le droit civil, avant tout un style ?, N. Kasirer (dir.), Les éditions Thémis, 2003, p. 101-120 ; M. Milet, Les professeurs de droit citoyens. Entre ordre juridique et espace public, contribution à l'étude des interactions entre les débats et les engagements des juristes français (1914-1995), thèse science politique, Paris II, 2000 ; G. Sacriste, Le droit de la République (1870-1914) : légitimation(s) de l'État et construction du rôle de professeur de droit constitutionnel, thèse science politique, Paris 1 , 2002 ; J.-L. Halpérin, « Présentation », Le Code civil 1804-1904. Livre du centenaire, rééd. 2004, Paris, Dalloz, p. 1-6 ; D. Bureau, « Les regards doctrinaux sur le Code civil », dans 1804-2004. Le Code civil. Un passé, un présent, un avenir, Dalloz, 2004, p. 171-210 ; D. Deroussin (dir.), Le renouvellement des sciences sociales et juridiques sous la III ${ }^{e}$ République. La faculté de Droit de Lyon, Paris, La mémoire du droit, 2007 ; F. Audren, « La Belle Époque des juristes catholiques (1880-1914) », Revue française d'histoire des idées politiques, 28, Juristes catholiques, 2008, p. 233-271 ; N. Hakim et F. Melleray, «La Belle Époque de la pensée juridique française », Le renouveau de la doctrine française. Les grands auteurs de la pensée juridique au tournant du $\mathrm{xx}^{e}$ siècle, Paris, Dalloz, 2009, p. 1-12 ; F. Audren, « Les professeurs de droit, la République et le nouvel esprit juridique. Introduction », Mil neuf cent. Revue d'histoire intellectuelle, 29, La Belle Époque des juristes. Enseigner le droit dans la République, 2011, p. 7-33 ; F. Audren et J.-L. Halpérin, La culture juridique française. Entre mythes et réalités ( $\mathrm{xIx}^{e}-\mathrm{xx}^{e}$ siècles), Paris, éd. du CNRS, 2013, not. p. 111-155.

18 Christophe Jamin note ainsi : « Gény contribue en définitive, fût-ce parfois à son corps défendant et dans une perspective résolument conservatrice, au même projet républicain que la plupart de ses contemporains en

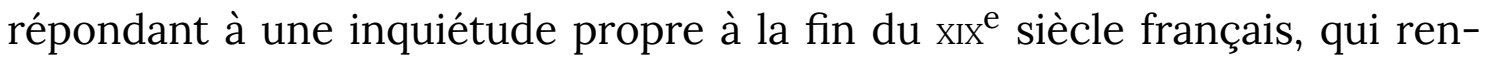
voie tout autant si ce n'est plus aux idéaux révolutionnaires et à leur nécessaire régénération qu'à la crainte du socialisme. » («François Gény d'un siècle à l'autre », François Gény, mythe et réalités, op. cit., p. 13). 
19 Sur Maurice Hauriou, cf. F. Audren et M. Milet, « Maurice Hauriou sociologue. Entre sociologue catholique et physique sociale », préface aux Écrits sociologiques de Maurice Hauriou, Dalloz, 2008, p. v-LVII ; N. Foulquier, « Maurice Hauriou, constitutionnaliste 1856-1929 », Le renouveau de la doctrine française, op. cit., p. 281-306 et récemment J.-M. Blanquer et M. Milet, L'invention de l'État. Léon Duguit et Maurice Hauriou et la naissance du droit public moderne, Paris, Odile Jacob, 2015.

20 Pour Duguit, outre l'ouvrage ci-dessus, cf. not. F. Melleray (dir.), Autour de Léon Duguit, Bruxelles, Bruylant, 2011 et, du même, « Léon Duguit. L'État détrôné », Le renouveau de la doctrine française, op. cit., p. 215-262.

21 Pour une bibliographie relative à Lambert, nous nous permettons de renvoyer à notre étude, Le droit civil et la législation ouvrière, Paris, Dalloz, coll. tiré-à-part, 2013, p. 35-37.

22 Cf. la stimulante étude de S. Bloquet, «Quand la science du droit s'est convertie au positivisme », Revue trimestrielle de droit civil, 2015, 1, p. 59-84.

23 Sur ce point, cf. O. Motte, Lettres inédites de juristes français du XIX ${ }^{e}$ siècle conservées dans les archives et bibliothèques allemandes, Bonn, 1989, volume 1, p. 752-755.

24 Le début de Science et technique est on ne peut plus explicite : «De quelle façon, jurisconsultes, travaillons-nous en fait ? De quelle façon devons-nous travailler ? Voilà bien, ce semble, traduit sous sa forme la plus simple, ce dont il nous importe, avant tout, de prendre conscience, et qui ne peut être fixé que par l'adaptation aux matières juridiques d'une théorie rationnelle, qui, partie des bases fondamentales de la connaissance, pénètre jusqu'aux ressorts de l'action. » (Science et technique, op. cit., tome $1, \mathrm{n}^{\mathrm{o}} 1$ ).

25 «François Gény et la renaissance du droit naturel », Le centenaire du doyen François Gény. Recueil des conférences prononcées les 26 et 27 octobre 1962, Paris, 1963, p. 39-56 ; Philosophie du droit, Paris, $2^{\mathrm{e}}$ éd., 1978, tome 2, $\mathrm{n}^{\mathrm{O}} 138$, p. 20 ; Leçons d'histoire de la philosophie du droit, nouvelle éd., Paris, 1962, p. 91. À compléter par N. Dissaux, op. cit., p. 417-429.

26 Cf. not. F. Audren, « La Belle Époque des juristes catholiques (18801914) », Revue française d'histoire des idées politiques, 28, Juristes catholiques, 2008, p. 233-271 ; M. Tancelin, « Pour en finir avec le mythe de la modernité de Gény », François Gény, mythe et réalités, op. cit., p. 373-380. D’un point de vue plus large, cf. C. M. Herrera, Les juristes face au politique. Le droit, la gauche, la doctrine sous la III ${ }^{e}$ République, Paris, Kimé, 2003. Pour des témoignages directs sur Gény, cf. M. Auberger, «Un autre regard sur François 
Gény et son œuvre », op. cit., p. 261-279 ainsi que, du même auteur, « La vie de François Gény : la doctrine et son époque », La pensée de François Gény, op. cit., p. 7-22. Christophe Jamin cite quant à lui une lettre explicite adressée à Saleilles par Gény le 27 septembre 1896 : «quoiqu'on en puisse dire, [le critère décisif] ne peut venir que de très-haut. Et, pour ma part, je ne puis le trouver ailleurs que dans la fin totale de l'humanité. Ce qui nous ramène bon gré mal gré, à un postulat métaphysique, sans lequel, toute science sociale reste vouée au pur empirisme » («François Gény d'un siècle à l'autre », op. cit., p. 18). Sur la famille Gény et son membre le plus célèbre (qui n'est donc pas François), cf. P. Artières, Vie et mort de Paul Gény, Paris, Le Seuil, 2013.

27 «Préface », Méthode d'interprétation, op. cit., tome 1, p. XIII-XIV.

28 À titre d'exemple, cf. dès le début de Science et technique : « j'entends m'expliquer en toute franchise, et prendre le bien et le vrai où ils m'apparaîtront, insoucieux des routines comme du parti pris. Pareille affirmation n'est peut-être pas inutile au seuil d'un travail, que je sens, bon gré mal gré, chargé de postulats métaphysiques et de jugements de laveur, entre lesquels le choix nécessaire pourra heurter certaines opinions courantes. Si je ne puis, plus que tout homme au monde, prétendre me soustraire à l'influence d'idées préconçues, de préjugés d'origine, de milieu ou d'intérêt, je m'efforcerai d'en réduire la portée par une entière bonne foi et une ferme volonté d'impartialité objective. Mais je ne vois pas pourquoi je renoncerais, par une sorte de snobisme scientifique, à des convictions réfléchies ou aux opinions qui, sur les points les plus obscurs de l'énigme de notre univers, me paraissent encore, jusqu'à nouvelle démonstration, les plus plausibles. D'autre part, sans céder à un engouement irraisonné, je me tiendrais pour coupable de lâcheté ou d'inertie intellectuelles, si j'hésitais à emprunter aux mouvements des idées, les plus riches et les plus osés de notre temps, les éléments sains et vrais que j’y croirai découvrir, pour en fortifier, en élargir, en accroître nos conceptions traditionnelles » (op. cit., tome 1, no 6, p. 18-19).

29 F. Gény, « La notion de Droit en France. Son état présent. Son avenir », Archives de philosophie du droit et de sociologie juridique, 1-2, 1932, n 2, p. 11.

30 Cf. Méthode d'interprétation, op. cit., tome 1, n os 98-105.

31 Sur ce renouveau, cf. not. D. Deroussin, «Une renaissance du droit naturel dans la doctrine civiliste à la Belle Époque : le droit naturel à contenu variable, le juge et le législateur », Un dialogue juridico-politique : le droit naturel, le législateur et le juge, Association française des historiens des idées politiques, Presses universitaires d'Aix-Marseille, 2010, p. 409-431 ; M.- 
Z. Zhu, Le droit naturel dans la doctrine civiliste de 1880 à 1940, thèse droit, Sciences Po, Paris, 2015.

32 Méthode d'interprétation, op. cit., tome 2, no 183.

33 Le construit, quant à lui, « au moyen d'un travail tout subjectif, [...] tend à ériger la règle brute en précepte capable de s'insérer dans la vie et d'animer celle-ci, en vue des fins suprêmes du droit » (Science et technique, op. cit., tome $\left.1, \mathrm{n}^{\mathrm{O}} 33\right)$.

34 Notons que ce donné réel est composé des « conditions de fait, où se trouve placée l'humanité »(sol, climat, productions, constitutions anatomiques et physiologiques, états psychologiques, sentiments religieux, économies) et que Gény n'hésite pas à naturaliser ces faits en prenant l'exemple des différences psychologiques ou morales entre mari et femme, lesquelles conduisent inéluctablement à des situations juridiques différentes. Il y a donc pour lui des éléments naturels à la base du droit positif dans chacun des donnés qui forment le socle du droit positif. Cf. Science et technique, op. cit., tome $2, \mathrm{n}^{\mathrm{O}} 167$.

35 Ce sont les faits et circonstances historiques ou autrement dit le « droit produit historiquement » (Science et technique, op. cit., tome 2, $\mathrm{n}^{\mathrm{o}} 168$ ).

36 Il s'agit des aspirations humaines (Science et technique, op. cit., tome 2, $\left.n^{0} 170\right)$.

37 Science et technique, op. cit., tome $4, \mathrm{n}^{\mathrm{O}} 302$.

38 Méthode d'interprétation, op. cit., tome 2, nº 159.

39 Notons d'ailleurs que Gény place la question du droit naturel au cœur du chapitre 9 de Science et technique intitulé : «Essai de synthèse des données du droit positif susceptibles de servir de bases à son élaboration scientifique » et que le sous-titre de la seconde partie de l'ouvrage consacrée à l'«Élaboration scientifique du droit positif » est « Lirréductible droit naturel ».

40 Cf. sa présentation dans La notion de droit positif à la veille du $\mathrm{xx}^{e}$ siècle. Discours prononcé à la séance solennelle de rentrée de l'université de Dijon le 8 novembre 1900, Dijon, 1900, not. p. 20.

41 Pour lui, l'analogie est le premier et principal recours de la libre recherche scientifique : « le fonctionnement de l'analogie consiste, tout entier, dans le procédé logique, qui vise à induire, de solutions particulières, le principe intime, qui les explique, pour chercher ensuite les conditions du même principe en d'autres hypothèses, et le leur appliquer par voie de dé 
duction » (Méthode d'interprétation, op. cit., tome 2, $\mathrm{n}^{\mathrm{o}} 165$ ). Elle permet de formuler une théorie d'ensemble devant être la « quintessence des solutions légales et traduisant l'esprit du droit positif moderne en une synthèse susceptible des plus précieuses applications pratiques » (Ibid., $\mathrm{n}^{\mathrm{0}} 167$ ).

42 Science et technique, op. cit., tome $3, \mathrm{n}^{\mathrm{O}} 181$.

43 Science et technique, op. cit., tome 3, $\mathrm{n}^{\mathrm{O}} 189$.

44 Notons que, dans une perspective positiviste, la «technique juridique » est un «Artefact humain qui peut avoir tous les contenus (y compris ceux que nous jugeons les plus tyranniques) » et qu'elle « est aussi et surtout un instrument de pouvoir » (J.-L. Halpérin, De la méthode historique en jurisprudence et de son avenir, Dalloz, 2013, p. 33). Cf. également, du même, « Le droit et ses histoires », Droit et société, 75, 2010, p. 295-313.

45 Cf. la réédition de son compte rendu de Gény ( Droit naturel et positivisme juridique », Annales de la Faculté de droit d'Aix, nouvelle série, 1, 1918, p. 3-47) : P. Jestaz, Droit naturel et positivisme juridique, Paris, Dalloz, 2013. Sur Ripert, cf. Cf. Anne Simonin, «La morale juridique de Georges Ripert », dans La République et son droit (1870-1930), A. Stora-Lamarre, J.-L. Halpérin et F. Audren (dir.), Besançon, PU de Franche-Comté, 2011, p. 359-379 ; J.L. Halpérin, Histoire du droit privé français depuis $1804,2^{\mathrm{e}}$ éd., Paris, PUF, Quadrige, 2012, no 127, p. 188-189. Sur le pseudo-positivisme de Ripert, cf. également M. Troper, «La doctrine et le positivisme (à propos d'un article de Danièle Lochak) », Les usages sociaux du droit, CURAPP-PUF, 1989, p. 286-292.

46 Science et technique, op. cit., tome 1, $\mathrm{n}^{\mathrm{0}}$ 11, p. 37.

47 Cf. not. «École historique et droit naturel d'après quelques ouvrages récents », Revue trimestrielle de droit civil, 1902, p. 80-112.

48 Méthode d'interprétation, op. cit., tome 2, nº 161.

49 Cf. N. Dissaux, op. cit., p. 417-429.

50 Gény définit la conscience comme le siège de l'idée de justice qui n'est autre que l'« esprit individuel de l'interprète » (Méthode d'interprétation, op. cit., tome $2, \mathrm{n}^{\mathrm{O}} 162$ ).

51 Méthode d'interprétation, op. cit., tome 2, nº 159.

52 Ibid., $\mathrm{n}^{\mathrm{0}} 160$.

53 Ibid., $\mathrm{n}^{\mathrm{0}} 161$.

54 Op. cit. et loc. cit. 
55 Science et technique, op. cit., tome $1, \mathrm{n}^{\mathrm{o}} 58$.

56 Ibid., $\mathrm{n}^{\mathrm{o}} 62$.

57 Cf. également son chapitre « La nécessité du droit naturel (Nouvel examen critique du positivisme juridique) 》(Science et technique, op. cit., tome $\left.4, \mathrm{n}^{\mathrm{o}} 213-265\right)$.

58 Science et technique, op. cit., tome $2, \mathrm{n}^{\mathrm{O}} 170$. Notons toutefois que la religion est l'élément central du donné idéal. Cf. également « La laïcité du droit naturel », Archives de philosophie du droit et de sociologique juridique, $\mathrm{n}^{\text {os }} 3$ 4, 1933, 12, p. 20 notamment.

59 Science et technique, op. cit., tome $2, \mathrm{n}^{\mathrm{o}} 169$.

60 La citation complète est limpide : « le donné rationnel résulte d'une élaboration véritablement scientifique, si l'on reconnaît ce caractère - comme on doit incontestablement le faire - à toute activité intellectuelle, pénétrant l'essence des choses par ses moyens propres de connaître, sans s'aider de forces plus obscures, dont la valeur paraît discutable » (Science et technique, op. cit., tome $2, \mathrm{n}^{\mathrm{0}} 169$ ).

61 Ibid., $\mathrm{n}^{\mathrm{o}} 171$.

62 Ibid., $\mathrm{n}^{\mathrm{0}} 172$.

63 Ibid., $\mathrm{n}^{\mathrm{0}} 162$.

64 Op. cit. et loc. cit.

65 Cf. C. Jamin, «François Gény d'un siècle à l'autre », François Gény, mythe et réalités, op. cit., p. 18-19 ; M.-Z. Zhu, op. cit., p. 121, p. 125 et p. 266-269.

66 Est éclairante sur ce point sa réplique à Georges Ripert au tout début du tome 4 de Science et technique, op. cit., p. vIII-IX.

67 Il affirme pourtant le contraire dans son article de 1933 « La laïcité du droit naturel », op. cit., p. 9. En réalité, il faut plutôt comprendre que l'aveu du contraire est impossible sans remettre en cause tout ce qu'il tente de faire depuis 1899, sans oublier que cela lui permet de se démarquer nettement de Georges Ripert et de Jean Dabin. Cf. « La notion de droit en France. Son état présent. Son avenir. Essai de ralliement autour d'un programme homogène d'études de philosophie du droit », Archives de philosophie droit et de sociologie juridique, $\mathrm{n}^{\mathrm{os}} 1-2,1931, \mathrm{n}^{\circ} 1, \mathrm{p} .9$. Non sans quelques incohérences, il écrit par ailleurs que « les idées, formant le fonds de notre morale religieuse, du point de vue chrétien [...] représentent un élément capital de notre civilisation » (Méthode d'interprétation, op. cit., tome 2, $\mathrm{n}^{\mathrm{O}}$ 167). 
68 Science et technique, op. cit., tome $2, \mathrm{n}^{\mathrm{o}} 164$.

69 La suite est intéressante en ce qu'il justifie le risque de subjectivité : «Je consens, qu'en passant à travers l'âme de chacun, les notions, objectives par essence, s'imprègnent de subjectivité, et que, n'apparaissant, dans la pratique, qu'adaptée aux éléments variables de la nature des choses positive, elles perdent, à des yeux peu clairvoyants leur aspects d'entités supérieures à l'homme et indépendantes de lui. » (Méthode d'interprétation, op. cit., tome $2, \mathrm{n}^{\mathrm{O}} 162$ ). Pour une comparaison intéressante, $\mathrm{cf}$. les propos apparemment similaires de Charles Beudant : «Il est une loi fondamentale, la droite raison, conforme à la nature, qui se révèle à chacun, constante et éternelle [...] Il n'est permis ni de l'abroger toute entière, ni d'y déroger en partie [...] ; elle n'a besoin de personne pour interprète ou organe : elle n'est pas autre à Rome ou à Athènes ; elle ne sera pas demain autre qu'aujourd'hui ; mais une, éternelle, immuable, elle dominera tous les peuples en tous les temps » (Le droit individuel et l'État. Introduction à l'étude du droit, Paris, Arthur Rousseau, 1891, no 19, p. 30-31).

70 Science et technique, op. cit., tome $2, \mathrm{n}^{\mathrm{o}} 70$.

71 Cf. par exemple ibid., $\mathrm{n}^{\mathrm{O}} 71$.

72 Ibid., no 169.

73 Science et technique, op. cit., tome $1, \mathrm{n}^{\mathrm{O}} 59$.

74 «Sans doute, à la rigueur, le législateur pourrait opérer lui-même les réformes. S'il les réalise, rien de mieux. Mais, vraiment, on ne peut se fier à lui, pour suffire à tout. Son intervention doit rester relativement rare, en matière de droit privé » (Méthode d'interprétation, op. cit., tome $2, \mathrm{n}^{\mathrm{O}} 185$ ).

75 Sur la part d'une certaine forme d'évolutionnisme dans les sciences sociales, cf. not. A. Testart, Avant l'histoire. L'évolution des sociétés de Lascaux à Carnac, Gallimard, Nrf, 2012, p. 46. Cf. également P. Veyne, Comment on écrit l'histoire, Le Seuil, 1996, p. 43 ; J.-L. Halpérin, De la méthode historique en jurisprudence et de son avenir, Dalloz, 2013, p. 29.

76 Sur ce point, cf. spécialement O. Jouanjan, «Présentation : Jhering ou l'amour du droit », R. von Jhering, La lutte pour le droit, Paris, Dalloz, 2006, p. v-XXXIII.

77 Méthode d'interprétation, op. cit., tome $2, \mathrm{n}^{\mathrm{O}} 164$. On ne peut manquer de relever ici l'hostilité farouche de Gény à la démocratie. L'opinion publique est même bannie du donné idéal qui doit pourtant permettre au juriste de connaître les aspirations humaines et l'« atmosphère ambiante ». Il note par 
exemple à propos du même donné idéal : «Il ne s'agit pas de l'opinion publique ; puissance aveugle et brutale, celle-ci peut parfois révéler l'idéal, mais reste incapable de le détruire, quand elle s'insurge contre lui sous la poussée d'un instinct évidemment inférieur » (ibid., $\mathrm{n}^{0}$ 170). «Sans parler de la difficulté, presque insurmontable en fait, d'une constatation convaincante de l'état de l'opinion publique sur une question de droit donnée, jestime que le jugement commun, tant qu'il n'est pas traduit en coutume caractérisée, n'a aucun titre à imposer son appréciation, pour la solution des problèmes juridiques ». Certes il doit reconnaitre que l'opinion publique est un fait social qui entre dans l'appréciation de l'interprète mais « cette opinion ne peut jouer le rôle d'une autorité pour l'interprète, ni lui servir de criterium pour discerner le droit positif à suivre, par cela même qu'elle ne saurait prétendre à représenter la vérité des choses, qu'il s'agit seule de découvrir et d'appliquer » (ibid., $\mathrm{n}^{0}$ 157). Son ami Saleilles, bien plus ouvert que le Nancéien, écrit quand à lui : «Pour construire solidement en matière juridique, il faut s'inspirer sans doute des sentiments et des instincts populaires, mais à condition de les transformer par la raison en assises profondément ancrées dans les esprits. Il faut confier à la logique de l'esprit ce qui vient de l'inspiration du cœur ; sinon on bâtit sur du sable » (Raymond Saleilles, «Droit civil et droit comparé », Revue internationale de l'enseignement, 61, 1911, p. 21). Cf. également son très explicite article « Le droit romain et la démocratie », Studi di diritto romano, di diritto moderno e di stroria del diritto publicati in onore di Vittorio Scialoja nel XXV anniversario del suo insegnamento, Ulrico Hoepli, Milan, 1905, volume 2, p. 713-729.

78 Sur ce point, cf. F. Audren, Les juristes et les mondes de la science sociale en France. Deux moments de la rencontre entre droit et science sociale au tournant $d u \mathrm{xIx}^{e}$ siècle et au tournant $d u \mathrm{xx}^{e}$ siècle, thèse droit Dijon, dactyl., 2005 ; « Comment la science sociale vient aux juristes? Les professeurs de droit lyonnais et les traditions de la science sociale (1875-1935) », Le renouvellement des sciences sociales sous la III République. La Faculté de droit de Lyon, D. Deroussin (dir.), Paris, La Mémoire du droit, 2007, p. 3-50. Cf. également A.-J. Arnaud, Les juristes face à la société $d u$ XIX $x^{e}$ siècle à nos jours, Paris, PUF, 1975, p. 124-125 ; M. C. Prémont, «François Gény et les enjeux de la responsabilité civile », François Gény, mythe et réalités, op. cit., p. 101-160 ; N. Pierre, «François Gény et la responsabilité civile : le droit-science et le sens de l'histoire », op. cit., p. 164 ; V. Champeil-Desplats, Méthodologies du droit et des sciences du droit, Dalloz, Méthodes du droit, 2014, nº 378, p. 230. Pour des analyses différentes, cf. C. Thomasset, «Épilogue : l'actualité de François Gény et de son œuvre », op. cit., p. 279-284 et plusieurs contribu- 
tions dans O. Cachard, Fr. X. Licari et Fr. Lormant, La pensée de François Gény, op. cit., 2013.

79 Cf. les analyses de M.-Z. Zhu, op. cit., not. p. 63-68 et, pour une utile comparaison avec la prise en compte par les juristes de la jurisprudence et du phénomène contentieux, P.-N. Barénot, Entre théorie et pratique : les recueils de jurisprudence miroirs de la pensée juridique française (1789-1914), thèse droit, Bordeaux, 2014.

80 Cf. par exemple : « le droit reste homogène par la vertu même de la puissance mystérieuse, qui réunit constamment les éléments, issus de la nature des choses, aux inventions et constructions, dénotant la part d'artifice due aux efforts propres de l'homme, qui cherche à réaliser pleinement la justice » (Science et technique, op. cit., tome 3, no 268).

81 Science et technique, op. cit., tome $1, \mathrm{n}^{\mathrm{O}} 18$.

82 Ibid., n' 21.

83 Méthode d'interprétation, op. cit., tome 2, nº 164.

84 Ibid., $\mathrm{n}^{\mathrm{O}} 167$.

85 Ibid., no 168.

86 Ibid., $\mathrm{n}^{\mathrm{O}} 191$.

87 Pour une comparaison avec l'analyse tout à fait similaire de Bufnoir, cf. N. Hakim, « De l'esprit et de la méthode des civilistes de la seconde moitié du XIX ${ }^{\mathrm{e}}$ siècle : l'exemplarité de Claude Bufnoir », Droits, Revue française de théorie, de philosophie et de culture juridiques, 47, 2008, p. 45-75. Cf. également J. Brèthe de la Gressaye et M. Laborde-Lacoste, Introduction générale à l'étude du droit, Paris, Sirey, 1947, nº 126, p. 100.

88 Méthode d'interprétation, op. cit., tome 2, no 191. Il relève spécialement l'apport en la matière de Paul Gide, Charles Lefebvre, Paul Viollet, Philippe Sagnac et Charles Brissaud.

89 Résumant la pensée de Saleilles en la matière, il écrit : « la méthode historique ne consiste pas tant, à découvrir dans le passé, des expériences toutes faites, que le présent dût s'assimiler comme telles, mais, surtout, à tenir compte de l'évolution constante du monde, pour mesurer la réglementation, actuellement opportune, dans l'état présent de nos besoins et de nos ressources, en ne demandant à l'histoire, proprement dite, que l'enseignement général, procuré par l'exemple des adaptations les mieux réussies » (Méthode d'interprétation, op. cit., tome 2, no 191). 
90 Op. cit. et loc. cit.

91 Ibid., no 194.

92 Ibid., nº 138.

93 Ibid., nº 142.

94 Ibid., nº 143.

95 Ibid., $\mathrm{n}^{\mathrm{O}}$ 143. Il écrit plus loin : «L'autorité et la tradition ne constituent pas, à côté de la loi et de la coutume, une source formelle, indépendante et sui generis, de droit privé positif » (ibid., $\mathrm{n}^{0} 154$ ).

96 Ibid., $\mathrm{n}^{\mathrm{O}} 138$.

97 Ibid., no 139.

98 Op. cit. et loc. cit.

99 Sur ce point, cf. les remarques éclairantes de Natalino Irti (Le nihilisme juridique, Paris, Dalloz, Rivages du droit, à paraître).

100 Méthode d'interprétation, op. cit., tome 2, 1919, nº 167.

101 P. Veyne, Comment on écrit l'histoire, Le Seuil, 1996, p. 97.

102 Op. cit. et loc. cit.

103 Sur ce point, cf. J.-L. Halpérin, De la méthode historique en jurisprudence et de son avenir, Paris, Dalloz, 2013, p. 32.

104 Cf. J.-L. Halpérin, « Le droit et ses histoires », Droit et société, 75, 2010, p. 295-313 ; F. Audren et J.-L. Halpérin, La culture juridique française. Entre mythes et réalités (XIX ${ }^{e}-\mathrm{xx}^{e}$ siècles), Paris, éd. du CNRS, 2013, p. 186.

105 On pense bien entendu ici aux belles analyses de Hans Kelsen, notamment dans l'édition et la traduction récente de Qu'est-ce que la Justice, suivi de Droit et morale, préface de V. Lasserre, Genève, Markus Haller, 2012 (éditions originales autrichiennes de 1953 et 1960). Sur cette question entendue largement, cf. spécialement O. Jouanjan (dir.), Hans Kelsen. Forme du droit et politique de l'autonomie, Paris, PUF, 2010.

106 Sur l'historiographie de l'histoire du droit, cf. J. Poumarède, « Pavane pour une histoire du droit défunte (sur un centenaire oublié) », Procès. Cahiers d'analyse politique et juridique, 6, 1980, p. 91-102 ; J.-L. Halpérin, "L'histoire du droit constituée en discipline : consécration ou repli identitaire ? », Revue d'histoire des sciences humaines, 4, 2001, p. 9-32 ; F. Audren, «L'histoire à contre-courant. Discipline et indiscipline dans la section d'histoire du droit (1970-1990) », Le droit en révolutions(s). Regards sur la critique 
du droit des années 1970 à nos jours, X. Dupré de Boulois et M. Kaluszynski (dir.), Paris, LGDJ-Lextenso éditions, 2011, p. 51-69 ; P. Bonin, « L'historiographie de l'histoire du droit, tendance récente et prochains territoires », L'Histoire du droit en France. Nouvelles tendances, nouveaux territoires, B. d'Alteroche et J. Krynen (dir.), Paris, Garnier, 2014, p. 533-558.

\section{RÉSUMÉS}

\section{Français}

La présente étude entend examiner la relation qu'entretient François Gény à l'histoire du droit. Une lecture contextuelle de son œuvre conduit à remettre en question l'ouverture du doyen de Nancy aux sciences dites auxiliaires du droit et à recentrer le regard sur le cœur d'une pensée fermement arrimée à un droit naturel immuable et universel. L'histoire finit ainsi par être réduite à la tradition et à l'autorité ou, autrement dit, à une méthode dogmatique et axiologique.

\section{English}

The present study intends to examine François Gény's connection to history of Law. A contextual approach of his work leads to reconsider his opening to the auxiliary sciences of Law and to refocus on the heart of a though firmly fastened to an unchanging and universal natural law. The history is thus and finally reduced to tradition and to authority or, in other words, to a dogmatic and axiological method.

\section{INDEX}

\section{Mots-clés}

Gény François (1861-1959), droit naturel, histoire, histoire du droit, idéologie, tradition, autorité

\section{Keywords}

Gény François (1861-1959), natural law, history, legal history, ideology, tradition, authority

\section{AUTEUR}

\section{Nader Hakim}

Université de Bordeaux 